\title{
ESPR adopts British guidelines for imaging in suspected non-accidental injury as the European standard
}

\author{
Amaka C. Offiah • Catherine Adamsbaum • \\ Rick R. van Rijn
}

Received: 23 July 2014 / Accepted: 6 August 2014 /Published online: 7 October 2014

(C) Springer-Verlag Berlin Heidelberg 2014

National surveys conducted over the last decade [1-3] have highlighted inconsistencies among departments as regards the projections and quality of images obtained when child abuse is suspected. Has publication of the results of these surveys led to any improvement? We would like to think so, and while a follow-up UK audit demonstrated only a modest increase in adherence to the then-British Society of Paediatric Radiology guidelines [4], anecdotally we believe that the quality of skeletal surveys and therefore the accuracy and speed of diagnosis in these cases has significantly improved.

However, rather than repeating national surveys, under the leadership of Rick van Rijn (ex-chairperson of the ESPR (European Society of Paediatric Radiology) Child Abuse Taskforce), a Europe-wide survey of ESPR members was undertaken. The linked article in this edition of Pediatric Radiology [5] presents the findings.

Here, we would like to emphasize the key outcome that followed presentation of the results by Amaka Offiah to the Child Abuse Taskforce during the ESPR meeting in Budapest,

\footnotetext{
A. C. Offiah $(\bowtie)$

Academic Unit of Child Health, Stephenson Wing, Sheffield

Children's NHS Foundation Trust, WesternBank, Sheffield S10 2TH,

UK

e-mail: amaka.offiah@nhs.net

C. Adamsbaum

Faculty of Medicine, Paris Sud University, Le Kremlin Bicêtre, France

\section{Adamsbaum}

Pediatric Radiology Department, AP-HP, Bicêtre Hospital,

Le Kremlin Bicêtre, France

R. R. van Rijn

Department of Radiology, Emma Children's Hospital - Academic

Medical Center, Amsterdam, The Netherlands
}

Hungary, in June 2013, namely an overwhelming vote in favor of adopting the Royal College of Radiologists/Royal College of Paediatrics and Child Health (RCR/RCPCH) Guidelines for Imaging in Suspected Non-Accidental Injury [6] as the standard across Europe. We believe this will lead to improved detection of abuse in children and to more robust safeguarding protocols, and it is with great pleasure that we announce that the ESPR committee has ratified this move. The guidelines are available at http://www.rcr.ac.uk/docs/ radiology/pdf/RCPCH_RCR_final.pdf.

We are in the process of translating these guidelines into other major European languages, and would ask you, in your various countries and beyond, to please spread the word.

Conflicts of interest None

\section{References}

1. Offiah AC, Hall CM (2003) Observational study of skeletal surveys in non-accidental injury. Clin Radiol 58:702-705

2. Kleinman PL, Kleinman PK, Savageau JA (2004) Suspected infant abuse: radiographic skeletal survey practices in pediatric health care facilities. Radiology 233:477-485

3. Van Rijn RR, Kleviet N, Hoekstra R et al (2009) Radiology in suspected non-accidental injury: theory and practice in The Netherlands. Eur J Radiol 71:147-151

4. Swinson S, Tapp M, Brindley R et al (2008) An audit of skeletal surveys for suspected non-accidental injury following publication of the British Society of Paediatric Radiology guidelines. Clin Radiol 63: 651-656

5. Hulson O, van Rijn RR, Offiah AC (2014) Results of a European-wide survey regarding imaging in non-accidental injury: the need for and adoption of a consensus protocol. Pediatr Radiol [In press]

6. Royal College of Radiology, Royal College of Paediatrics and Child Health (2008) Standards for radiological investigations of suspected non-accidental injury. https://www.rcr.ac.uk/docs/radiology/pdf/ RCPCH_RCR_final.pdf. Accessed 4 Aug 2014 